\title{
Usefulness of step-by-step double-clip-with-line method in duodenal endoscopic submucosal dissection
}

\author{
Akira Yoshida ${ }^{a}$, Takuma Okamurab ${ }^{b}$, Tsutomu Kanda ${ }^{a}$, Hajime Isomoto ${ }^{a}$
}

Tottori University, Yonago; National Cancer Center Hospital, Tokyo, Japan

Duodenal endoscopic submucosal dissection (ESD) is difficult because of insufficient mucosal elevation and poor endoscopic maneuverability [1]. We performed successful ESD of a superficially extensive duodenal bulb tumor using the step-by-step double-clip-with-line method. The patient had a flat-elevated large lesion, $40 \mathrm{~mm}$ in diameter, at the greater curvature of the duodenal bulb (Fig. 1A), for which ESD was performed as previously described [2], using the same devices and electrosurgical generator settings. After circumferential mucosal incision (Fig. 1B), we attempted submucosal dissection, which was difficult because of inadequate submucosal elevation. Therefore, we selected the modified clip-with-line method (Video 1). The first endoclipwith-line (dental floss) was placed on the anterior side of the tumor mucosa, as an anchor [3] to insert the scope into the submucosal layer (Fig. 1C). After approximately half of the submucosal dissection had been completed, the second endoclip-with-line was placed on the posterior mucosal aspect of the tumor to secure a better submucosal space (Fig. 1D). Following placement of the second clip, and owing to the bilateral traction of the tumor mucosa parallel to the muscular layer plane, the space of the submucosal layer could be enlarged, extended, and observed (Fig. 2A). We dissected the tumor en bloc, accurately and safely (Fig. 2B). Complete closure was achieved without complications, using an endoloop and endoclip. Intramucosal adenocarcinoma with no lymphovascular invasion and negative margins was diagnosed.

ESD of large duodenal tumors is challenging. However, the step-by-step double-clip-with-line method is useful for improving endoscopic submucosal insertion. This method (you can se at: https://youtu.be/t8DPMAzbUms) can improve the management of similar duodenal ESD cases.

${ }^{a}$ Division of Medicine and Clinical Science Department of Multidisciplinary Internal Medicine Faculty of Medicine, Tottori University, Yonago (Akira Yoshida, Tsutomu Kanda, Hajime Isomoto);

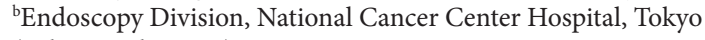
(Takuma Okamura), Japan

\section{Conflict of Interest: None}

Correspondence to: Akira Yoshida, MD, PhD, Division of Medicine and Clinical Science Department of Multidisciplinary Internal Medicine Faculty of Medicine, Tottori University, Yonago, 36-1 Nishicho, Yonago, 683-8504, Japan, e-mail: akirayoshida1021@yahoo.co.jp

Received 30 January 2021; accepted 6 March 2021; published online 27 May 2021

DOI: https://doi.org/10.20524/aog.2021.0629

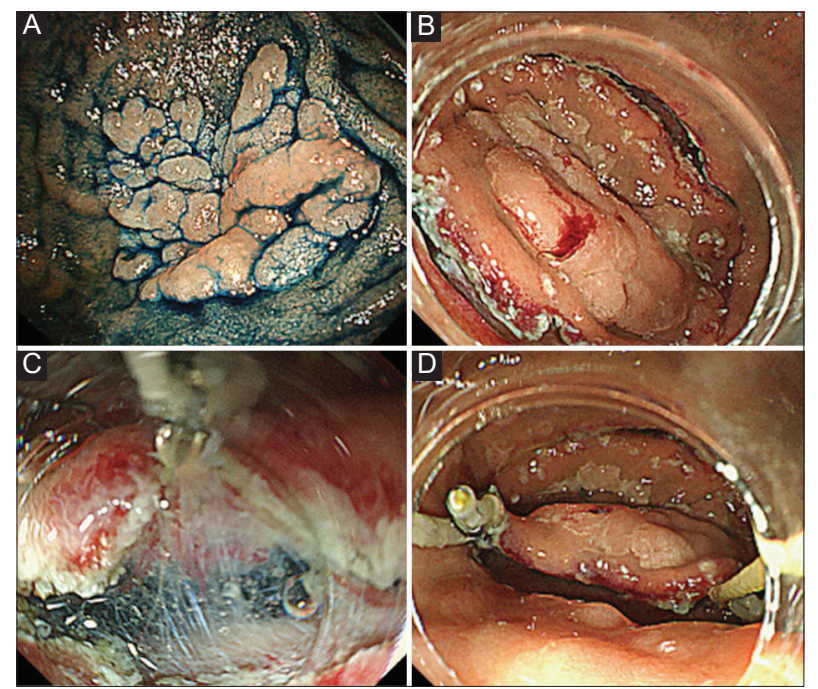

Figure 1 (A) A flat-elevated tumor, 40-mm in diameter, at the greater curvature of the duodenal bulb. (B) The mucosal incision of the tumor using a 2-mm flush knife (Flush knife BT-S; Fujifilm, Tokyo, Japan). (C) The first endoclip- (HX-610-135; Olympus Medical Systems Corp., Tokyo) with-line (dental floss) is placed on the anterior side of the tumor mucosa as an anchor. (D) The second endoclip-with-line is placed on the posterior mucosal aspect of the tumor mucosa
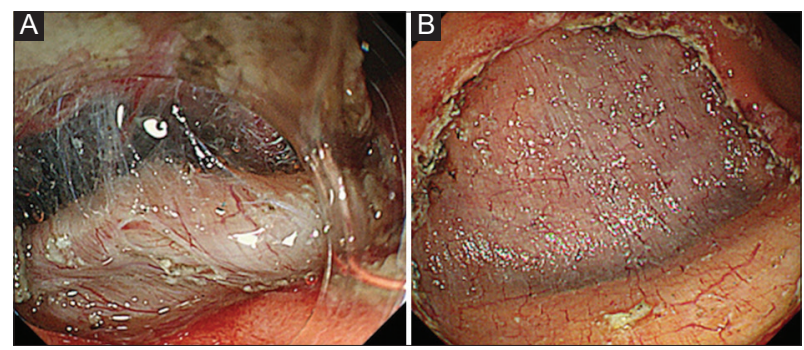

Figure 2 (A) After the second endoclip-with-line, we secured a better submucosal space. (B) The tumor is resected accurately and safely

\section{References}

1. Ochiai $Y$, Kato M, Kiguchi Y, et al. Current status and challenges of endoscopic treatments for duodenal tumors. Digestion 2019;99:21-26.

2. Yoshida $\mathrm{A}$, Kawaguchi $\mathrm{K}$, Yashima $\mathrm{K}$, Iwasaki $\mathrm{K}$, Isomoto $\mathrm{H}$. Endoscopic submucosal dissection for a laterally spreading tumor involving the colon diverticulum using a knife with water supply function. VideoGIE 2020;5:207-209.

3. Yamamoto K, Hayashi S, Saiki H, et al. Endoscopic submucosal dissection for large superficial colorectal tumors using the "clipflap method". Endoscopy 2015;47:262-265. 